Trauma Berufskrankh 2007 - 9 [Suppl 2]:S249-S256 DOI 10.1007/s10039-006-1149-4

Online publiziert: 10. Juni 2006

(c) Springer Medizin Verlag 2006
C. Rusu $\cdot$ L. Herold · C. Voigt · H. Lill

Klinik für Unfall- und Wiederherstellungschirurgie, Friederikenstift Hannover, Hannover

\section{Thorakolumbaler Übergang der Wirbelsäule}

Bedingt durch unzureichende Diagnostik, fehlende Erfahrung, nicht korrekte Indikationsstellung und durch Versagen operativer Therapien kann es zur Fehlverheilung oder Fehlstellung von Wirbelbrüchen, insbesondere am thorakolumbalen Übergang, kommen. Fehlstatik der Wirbelsäule, Schmerzen und Arbeitsunfähigkeit bis hin zu Erwerbsunfähigkeit der Betroffenen machen die Korrektureingriffe notwendig. Oft ist es erforderlich, bereits implantiertes Osteosynthesematerial zu entfernen und den Eingriff kombiniert dorsoventral zu instrumentieren. Um ein bestmögliches Ergebnis zu erzielen, sind eine korrekte Indikationsstellung, eine akribische präoperative Planung und eine hohe Erfahrung des Operateurs erforderlich. Da diese Voraussetzungen meist nur an spezialisierten Wirbelsäulenzentren anzutreffen sind, sollten Korrektureingriffe an der Wirbelsäule solchen Institutionen vorbehalten sein.

In der vorliegenden Arbeit sollen die Ursachen, die Indikation, die Durchführung und die Ergebnisse nach Korrektureingriffen am thorakolumbalen Übergang vorgestellt werden.

\section{Posttraumatische Fehlstellungen der Brust- und Lendenwirbelsäule}

Die Ursachen für Fehlbildungen der Wirbelsäule reichen von ausgeprägten Fehlstellungen, z. B. im Rahmen einer Grunderkrankung, wie der Tuberkulose, über degenerativ bedingte Fehlstellungen, bis hin zu posttraumatischen Fehlstellungen, welche im Folgenden vorgestellt werden.

Frakturen der Wirbelsäule betreffen am häufigsten den thorakolumbalen Übergang [2, 14], und unfallbedingte Deformitäten sind am häufigsten in diesem Abschnitt lokalisiert, wobei posttraumatische Fehlstellungen jeden Wirbelsäulenabschnitt betreffen können. Ihr Ausmaß reicht, in Abhängigkeit von der initialen Verletzung und der primären Therapie, von einfachen eindimensionalen Achsabweichungen, welche funktionell wenig wirksam sind, bis hin zu komplexen dreidimensionalen Verbiegungen der Wirbelsäule von äußerst invalidisierendem Charakter.

Klinisch symptomatische, von einem invalidisierenden Schmerz begleitete Deformitäten sind neben den radiologisch messbaren, meist kyphotischen Fehlstellungen die Indikationskriterien für einen Korrektureingriff. Dessen Ziel ist, die Schmerzen zu reduzieren, die Funktion und nicht zuletzt die Arbeitsfähigkeit wieder herzustellen.

\section{Ursachen posttraumatischer Fehlstellungen}

Dass posttraumatische Kyphosen am häufigsten im BWS-LWS-Übergang anzutreffen sind, hat seine Ursache in der spezifischen Belastungssituation des Übergangs von der relativ rigiden BWS zur deutlich mobileren LWS. Bei der Analyse der Fälle, die einer operativen Korrektur bedürfen, lassen sich in aller Re- gel die Gründe für den Misserfolg einer Therapie nach Frakturen in diesem Bereich aufdecken.

Die im Folgenden skizzierten Ursachen für ein unbefriedigendes Ergebnis können allein verantwortlich sein oder in Kombination auftreten, wobei sie sich in ihrer Wirkung verstärken:

- Bagatellisierung der Verletzung

- Fehlende oder verspätete Diagnostik

- Unzureichende Diagnostik

- Fehlinterpretation der Befunde

- Nicht korrekte Indikation zur konservativen Therapie

- Unzureichende Kontrolle nach konservativer Therapie

- Operationsassoziierte Ursachen

- Unzureichende Kontrolle nach operativer Therapie

Unzureichende bildgebende Diagnostik und das initiale Fehlen von subjektiven Beschwerden bei polytraumatisierten $\mathrm{Pa}$ tienten, beispielsweise durch Analgosedierung, können dazu führen, dass Wirbelsäulenverletzungen erst verspätet erkannt werden. Nicht nur deswegen sollte bei Mehrfachverletzten oder nach Hochrasanztraumen ein konsequentes Scanning durch die Traumaspirale erfolgen. Verletzungen, die sich der konventionellen Bildgebung entziehen, können so rasch diagnostiziert werden.

Eine konsequente Analyse der Frakturen und eine ebenso konsequente Klassifikation verringern die Gefahr einer Fehlinterpretation. Von der Vielzahl der möglichen Klassifikationen hat sich in un- 


\section{Wirbelsäule}

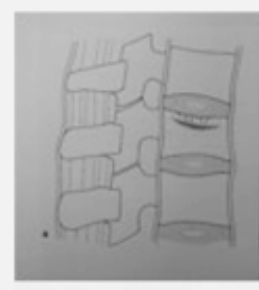

A 1.1

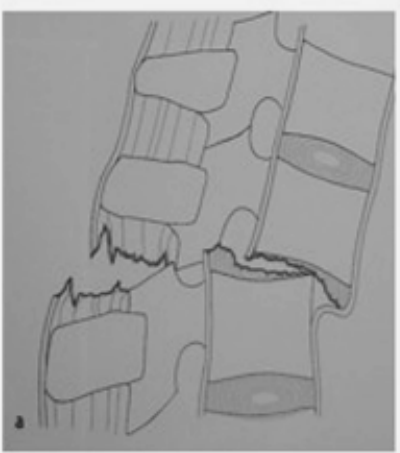

B 1.1.2

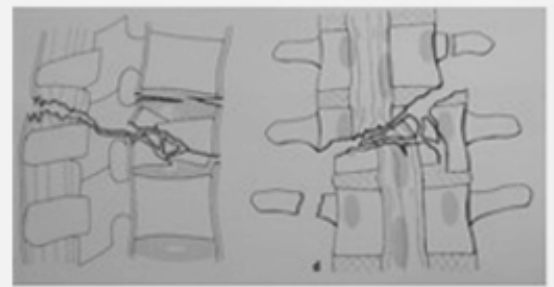

C 1.3

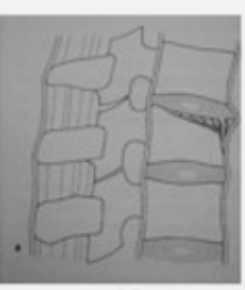

A 1.2.1

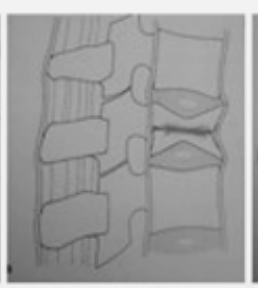

A 1.3

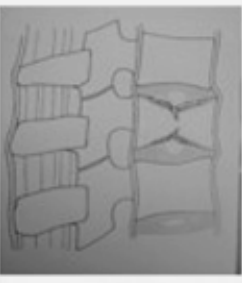

A 2

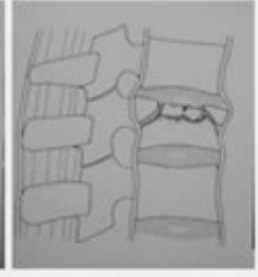

A 3.1.1

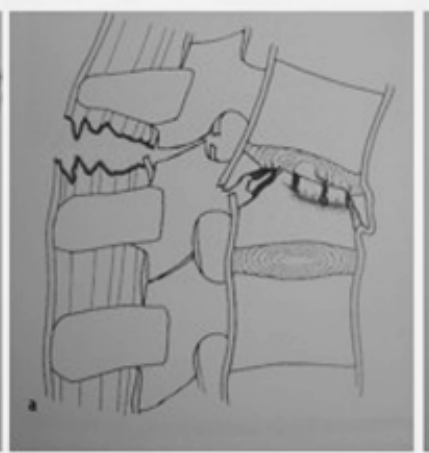

B 1.2.1

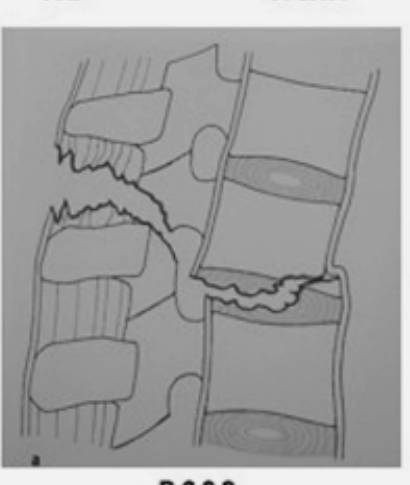

B 2.2.2

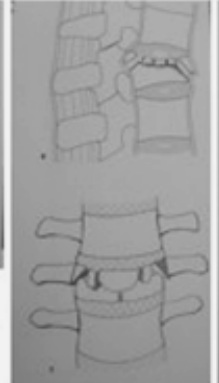

A 3.2 .1

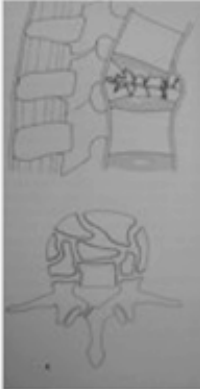

A 3.3 .2

\section{AO - Klassifikation}

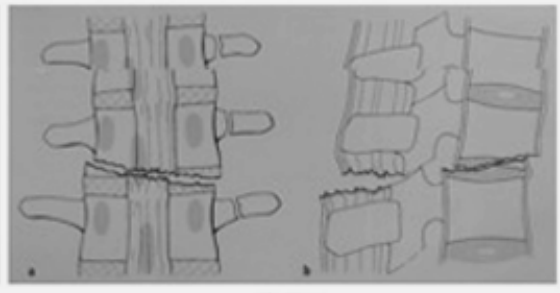

C 2.1

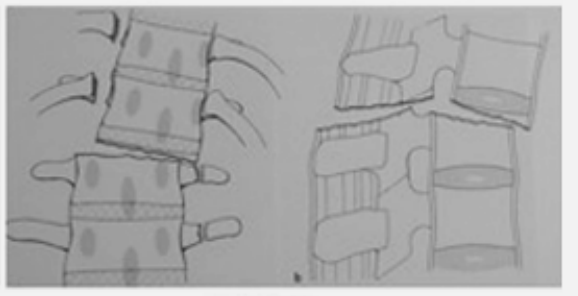

C 3.1

Abb. 1 A Schematische Beispiele der AO-Klassifikation von Wirbelbrüchen nach den Kriterien von Magerl et al., aus Blauth et al. [2]

serer Klinik diejenige nach den Kriterien der AO bewährt ( $\bullet$ Abb. 1).

Das Verkennen einer instabilen B1.2Verletzung und das Verwechseln mit einer stabilen A1-Verletzung führen zu einer inadäquaten Therapie und somit unabdingbar zur Ausbildung einer posttraumatischen Kyphose. Vaccaro u. Silber [19] beschrieben die Spezifität einer B1.2-Verletzung als „verdeckte Instabilität“.

\section{Indikation zur konservativen vs. operativen Therapie}

Die Indikation zur konservativen Therapie ist abhängig von der Stabilität und von der Höhenlokalisation der Fraktur. Stabile Brüche im thorakolumbalen Übergang (Typ A1.1-A2.2) ohne wesentliche Fehlstellung können frühfunktionell behandelt und müssen im Verlauf radiologisch kontrolliert werden $[2,8]$. Kneifzangen(Typ A2.3) und Berstungsbrüche (Тyp А3) gelten als instabil und sollten daher kombiniert dorsoventral, möglichst kurzstreckig, instrumentiert werden. In der un- teren LWS genügt häufig eine reine dorsale, zuggurtende Osteosynthese [8].

Brüche mit Beteiligung der hinteren Säulen (Typ B) sollten immer operativ versorgt werden, wobei die Entscheidung zum rein dorsalen oder kombiniert dorsoventralen Vorgehen in Abhängigkeit vom knöchernen Defekt des Wirbelkörpers zu treffen ist.

Hochgradig instabile Typ-C-Verletzungen werden in der Regel nach der Reposition kombiniert instrumentiert [2, 8]. Die Art des vorderen Zugangs (minimalinvasiv oder offen) ist von der Höhenlokalisation und der Erfahrung des Operateurs abhängig.

\section{Unzureichende Kontrolle nach konservativer Therapie}

Werden Frakturen konservativ behandelt, kommt einer adäquaten Kontrolle im Sinne von radiologischen Verlaufskontrollen eine entscheidende Bedeutung zu. Sei es eine korrekt eingeschätzte, initial stabile A1-Fraktur, die konservativ behan- delt wird, oder auch eine zunächst nicht korrekt eingeschätzte $\mathrm{A}_{3}$-Fraktur, können durch eine rechtzeitige Röntgenkontrolle das Versagen der konservativen Therapie erkannt und frühzeitig eine operative Stabilisierung durchgeführt und damit ein später Korrektureingriff vermieden werden.

Das initiale Trauma kann zu einer Schädigung des spongiösen Knochens, insbesondere im osteoporotischen Wirbelkörper, führen und somit eine sekundäre Knochennekrose auslösen, was trotz korrekter Indikation zum Versagen der konservativen Therapie führt [14]. Umso mehr wird deutlich, wie wichtig eine konsequente radiologische Verlaufskontrolle ist (• Abb. 2, 3).

\section{Operationsassoziierte Ursachen}

Häufig lässt sich eine posttraumatische Kyphose trotz operativer Stabilisierung antreffen, wenn aus unterschiedlichen Gründen zwar die technisch relativ einfache dorsale Stabilisierung durchgeführt 
wurde, die technisch anspruchsvollere Rekonstruktion der ventralen Säule aber unterblieb. Zahlreiche Arbeiten haben gezeigt, dass es nach alleiniger dorsaler Instrumentierung instabiler Frakturen in bis zu 63\% der Fälle zu signifikanten, teils revisionspflichtigen Korrekturverlusten kommt $[10,11,12]$.

Die nicht ausreichende Versorgung eines kompletten Berstungsbruchs des 12. BWK (AO-A3.3) durch eine bisegmentale, dorsale USS-Spondylodese ist in $\mathbf{A b b . 4}$ gezeigt. Zur Stabilitätsunterstützung wurde die 43-jährige Patientin wochenlang in einem Softcast-Korsett „ruhig gestellt“. Wegen fehlender ventraler Abstützung kam es sekundär zum Bruch des Osteosynthesematerials und zur Ausbildung einer posttraumatischen Kyphose.

Die Zahl der Faktoren, die trotz Operation zu einer posttraumatischen Kyphose führen, ist groß: So können ausgedehnte Laminektomien im Rahmen neurologischer Defizite zu Instabilität und damit zur Fehlstellung führen. Fehlplatzierung von Pedikelschrauben kann einen Schraubenausbruch und damit ebenfalls ein Implantatversagen zur Folge haben (- Abb.5). Auch bei primär richtig erkannten und gleich dorsoventral kombiniert instrumentierten Frakturstabilisierungen kann es zum Repositionsverlust und zur Fehlstellung des betroffenen Segments kommen, z. B. durch Fehlplatzierung eines Knochenspans, Spannekrose, Pseudarthrosenbildung, Fehllage eines Cages (primär oder sekundär durch Einsinken in den benachbarten Wirbelkörper).

\section{Korrekturspondylodese}

\section{Indikationen}

\section{Klinische Symptomatik}

Unabhängig von mess- oder sichtbaren Achsfehlstellungen und dem radiologischen Befund ist die Klinik zur Indikationsstellung immer in den Vordergrund zu stellen. Bei fehlenden klinischen Symptomen und deutlichen Achsfehlstellungen sollte der Patient auf die möglichen Spätfolgen bei fortbestehender Deformität hingewiesen werden.

Bereits die einfache Betrachtung des entkleideten Verletzten ermöglicht eine Einschätzung des Ausmaßes der funktionell beeinträchtigenden Wirbelsäulenfehl-

Trauma Berufskrankh 2007 - 9 [Suppl 2]:S249-S256 DOI 10.1007/s10039-006-1149-4

(c) Springer Medizin Verlag 2006

C. Rusu $\cdot$ L. Herold $\cdot$ C. Voigt $\cdot$ H. Lill

Thorakolumbaler Übergang der Wirbelsäule. Behandlung posttraumatischer Fehlstellungen

\section{Zusammenfassung}

In der überwiegenden Anzahl der Patienten mit posttraumatischen Fehlbildungen der Wirbelsäule geben Schmerzen Anlass zu einem Korrektureingriff. Die Ursachen der posttraumatischen Fehlstellung sind zahlreich, häufig handelt es sich jedoch um die nicht korrekte Indikationsstellung zur konservativen Therapie oder die unzureichende operative Versorgung (nur dorsal instrumentiert). Korrektureingriffe bedürfen einer korrekten Indikationsstellung und einer präzisen präoperativen Planung. Der Eingriff selbst wird einzeitig, dorsoventral kombiniert ins-

\section{Thoracolumbar spine. Treatment of posttraumatic deformity}

\section{Abstract}

Most patients with painful posttraumatic spinal deformity need reconstructive surgery. There are many reasons for such deformity; often it has developed because the indications for conservative therapy were not correctly observed or an operative treatment was inadequate (dorsal instrumentation only). Corrective surgery requires strict observation of the indications and precise preoperative planning. The instrumentation for the intervention is dorsoventral, and the intervention must be completed in a single session, trumentiert, wobei die ventrale Fusion minimalinvasiv, endoskopisch assistiert, durchgeführt werden kann. Aufgrund des technisch anspruchsvollen Eingriffs und des Operationsrisikos sollten Korrektureingriffe nur von erfahrenen Chirurgen an Wirbelsäulenzentren durchgeführt werden.

\section{Schlüsselwörter}

Posttraumatische Kyphose · Korrekturspondylodese - Thorakoskopie - Thorakolumbaler Übergang · Wirbelsäulenzentrum though the ventral fusion can be performed as a minimally invasive, endoscopically assisted technique. Because the procedure is difficult and the surgery involves a high level of risk, reconstructive surgery of the spine should be practised only by experienced surgeons working in spine centres.

\section{Keywords}

Posttraumatic kyphosis - Reconstructive spondylodesis - Thoracoscopy - Thoracolumbar spine $\cdot$ Spine centre 

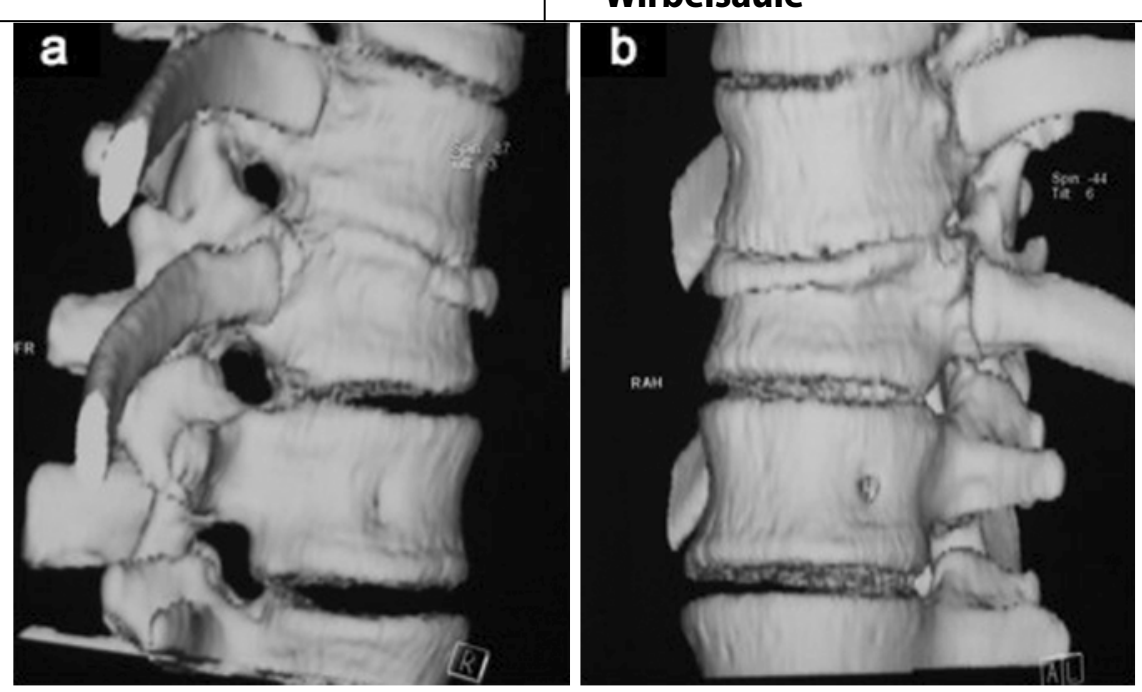

Abb. 2a,b $\Delta$ Posttraumatische Kyphose, 17 Jahre alter männlicher Patient, 17,5 Monate nach Unfall (inkompletter kranialer Berstungsbruch Th12 - AO A3.1.1) bei konservativer Therapie, Grund-Deckplatten-Winkel (GDW) $25^{\circ}$
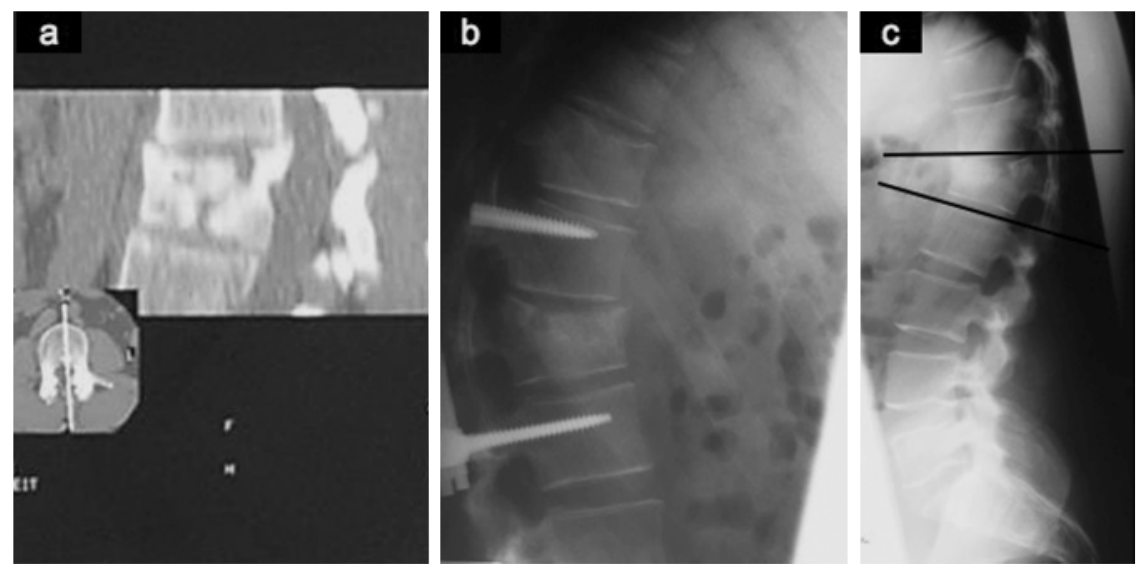

Abb. 3a-c $\boldsymbol{\Delta} 32$ Jahre alter männlicher Patient, 19,5 Monate nach Unfall (LWK1-Pincer-Fraktur - AO A2.3) nach alleiniger dorsaler Instrumentierung, Materialbruch und Materialentfernung, GDW $30^{\circ}$

stellung. Der Rippenbuckel weist auf Rotationsfehler, die Abweichung der Dornfortsätze aus der Mittellinie, der Schultertiefstand und die ungleichen Taillendreiecke auf Achsabweichungen in der Frontalebene und der Rundrücken mit Gibbusbildung auf Achsabweichungen in der Sagittalebene hin.

Die Ursache der Schmerzsymptomatik bei posttraumatischer Deformität der Wirbelsäule kann im Fall einer lokalen Instabilität diese segmentale Hypermobilität per se sein, im Fall einer rigiden Fehlbildung (mit oder ohne Spondylodese) ist es die kompensatorische Statikveränderung der angrenzenden Wirbelsäulensegmente, die v. a. in den kranial angrenzenden Abschnitten zu einer Mehr- oder Fehlbelastung führt $[7,13]$. Diese sekundäre Wirbelsäulenfehlstatik führt zu schmerz- haften muskulären Verspannungen und zur vorzeitigen osteochondralen Degeneration der benachbarten Segmente sowie zu Schäden im Bereich der angrenzenden Bandscheiben.

Hierbei ist eine Differenzierung zwischen vertebrogener und neurogener Schmerzursache, z. B. durch Affektion einer Nervenwurzel, wünschenswert und sollte durch eine gezielte neurologische Untersuchung präoperativ abgeklärt werden. Entscheidende präoperative Planungsschritte und ggf. weiterführende Bildgebung hängen hiervon $a b$.

\section{Neurologie}

Motorische oder sensible Defizite sowie Störungen der Blasen- und Mastdarmfunktion stellen eine Indikation zur Korrektur der Deformität, unter Dekompres- sion der betroffenen Strukturen, dar [1, 5]. Die Restitutionsprognose der neurologischen Defizite hängt von der Art und Dauer der Schädigung und dem Ausmaß der Ausfälle ab. Es konnte jedoch gezeigt werden, dass die Entlastung komprimierter neuraler Strukturen auch noch nach Jahren zu einer Erholung von Ausfallserscheinungen, sowohl von Rückenmark als auch von Cauda equina, führt $[4,5,15]$. Marré [14] hat dieser Liste von Kriterien aus seiner Erfahrung im eigenen Patientenkollektiv die medikamentös nicht beherrschbare Spastik im Fall einer kyphosebedingten Kompromittierung des Rückenmarks hinzugefügt.

\section{Achsabweichungen}

Fehlstellungen der Wirbelsäule in der frontalen und sagittalen Ebene sowie Rotationsfehlstellungen entwickeln sich sukzessive bei Versagen der initialen Therapie. Regelrechte Listhesen sind dagegen seltener anzutreffen. Im radiologischen a.-p. und seitlichen Strahlengang lassen sich Deformitäten am einfachsten mit dem so genannten Grund-DeckplattenWinkel (GDW), gemessen zwischen der Ebene der Grundplatte des kranial angrenzenden Winkels und der Deckplattenebene des kaudal angrenzenden Winkels, bestimmen (- Abb. 6). Eine deutliche Zunahme einer Kyphose oder Skoliose im Verlauf stellt eine Operationsindikation dar.

Böhler [3] beschrieb bereits 1972, dass ein Kyphosewinkel von $15^{\circ}$ regelmäßig zu klinischen Beschwerden führt. In der Literatur wird der Kyphosegrad für die Indikation zur Korrekturspondylodese unterschiedlich angegeben und variiert zwischen $15^{\circ}$ und $30^{\circ}[7,8,18]$. Allgemein kann jedoch davon ausgegangen werden, dass ein $G D W>20^{\circ} z u$ einer erhöhten Sekundärmorbidität führt und somit als Operationsindikation für den Korrektureingriff angesehen werden kann.

Häufig liegt eine Kombination verschiedener Fehlstellungen vor, was bei der Planung und Durchführung der Korrektur zu berücksichtigen ist.

\section{Ziel}

Durch Verbesserung der Wirbelsäulenstatik, Beseitigung von Instabilitäten und 

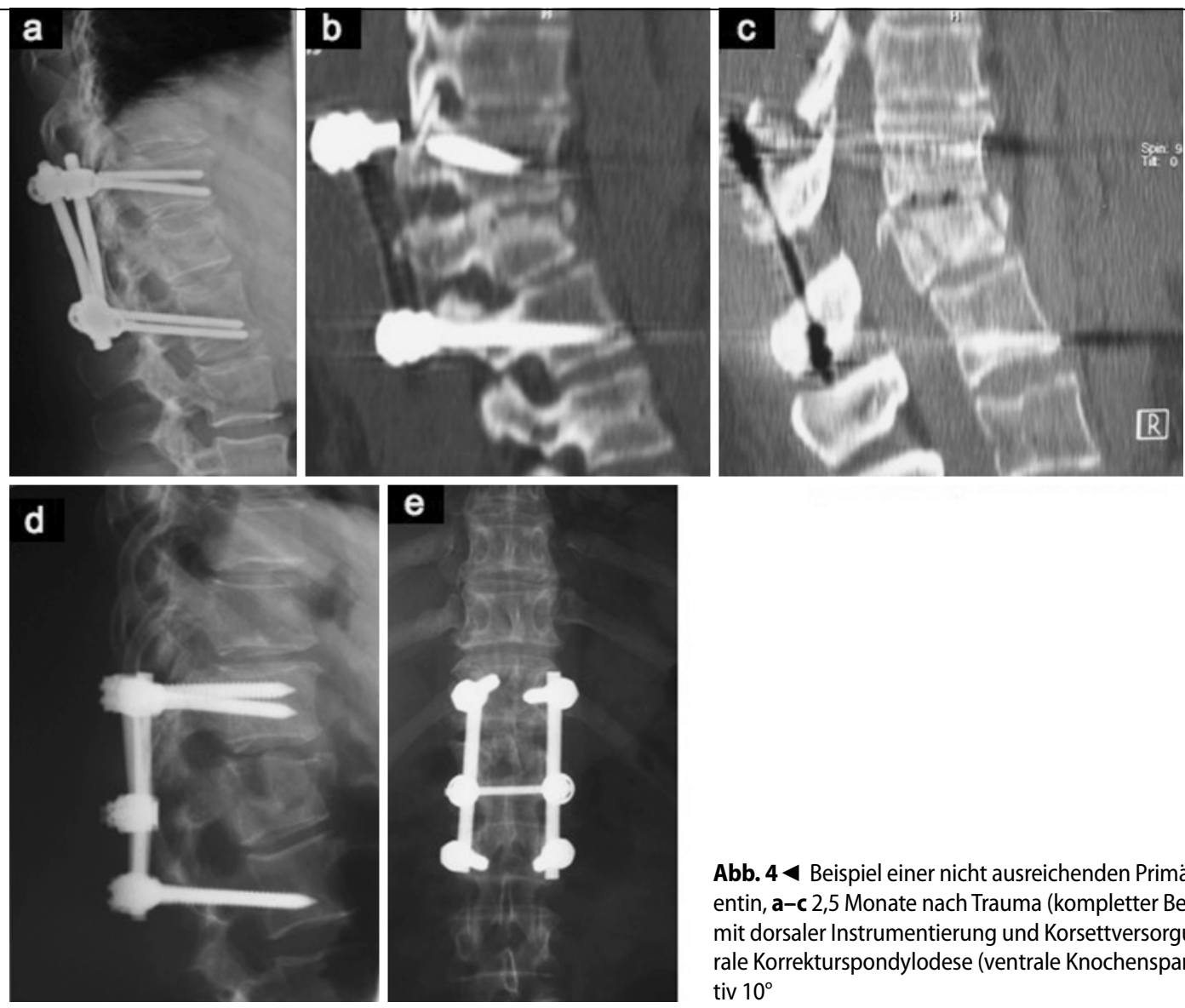

Abb. 44 Beispiel einer nicht ausreichenden Primärtherapie: 43 Jahre alte Patientin, a-c 2,5 Monate nach Trauma (kompletter Berstungsbruch Th12 - AO A3.3) mit dorsaler Instrumentierung und Korsettversorgung, GDW $16^{\circ}, \mathbf{d}, \mathbf{e}$ dorsoventrale Korrekturspondylodese (ventrale Knochenspaneinlage), GDW postoperativ $10^{\circ}$

die Dekompression neurogener Strukturen sollen

- eine Schmerzreduktion,

- eine Verbesserung der Wirbelsäulenbelastbarkeit und damit

- ein Gewinn an Lebensqualität

erzielt werden. Nicht zuletzt sollen Spätschäden vermieden und die Arbeitsfähigkeit des Patienten wiederhergestellt werden.

\section{Operative Besonderheiten}

Der Eingriff beinhaltet einen großen logistischen Aufwand. Für eine erfolgreiche Operation kann evtl. eine enge interdisziplinäre Zusammenarbeit mit anderen Fachabteilungen (Neurochirurgie, Thoraxchirurgie) notwendig werden.

\section{Planung}

Grundlage des operativen Vorgehens ist die umfassende Information über das individuell vorliegende Krankheitsbild. Hierzu wird umfangreiches Bildmaterial benötigt. Neben konventionellen Rönt- genaufnahmen der BWS und LWS in 2 Ebenen ist ein präoperatives CT mit $2 \mathrm{D}$ und 3 D-Rekonstruktion zur Planung des Vorgehens erforderlich. Ein MRT ist zur Beurteilung der umgebenden Weichteile (Bandscheibe, Bänder und neurogene Strukturen wie Rückenmark/Kauda/Nervenwurzel) unabdingbar. Notwendig wird dies v. a. bei neurologischen Defiziten, bedingt durch radikuläre Engpässe, und bei vorausgegangener Laminektomine. Bei einliegendem Osteosynthesematerial kann die detaillierte Beurteilbarkeit eines MRT aufgrund von Suszeptibilitätsartefakten nur eingeschränkt möglich sein.

Im konventionellen Röntgenbild ist eine exakte Vermessung der Achsabweichungen in der frontalen und sagittalen Ebene anhand des GDW notwenig, um die lokale und segmentale Kyphose- und oder Skoliosebildung zu quantifizieren. Seitverschiebungen der Wirbelkörper gegeneinander werden in der frontalen und der sagittalen Ebene durch Quotientenbildung des Versatzes und der kaudalen Deckplattengröße quantifiziert (• Abb. 6) [11].
$\mathrm{Zu}$ berücksichtigen ist auch, dass es sich bei vorausgegangenen, fehlgeschlagenen Operationen um Zustände handelt, die meist mehrere Monate, wenn nicht mehrere Jahre bestehen und dass durch Ausbildung von Vernarbungen und Kontrakturen eine einfache Reposition der Fehlstellung u. U. nicht oder zumindest nur sehr schwer möglich sein kann. Bei Fehllage des Osteosynthesematerials ist u. U. ein Wechsel des dorsalen Fixateurs notwendig, was bei Osteolysen der Pedikel zu erheblichen Schwierigkeiten bei der Neuplatzierung der Pedikelschrauben führen kann. In manchen Fällen kann es notwendig werden, ein weiteres Segment von dorsal zu erfassen. Aufgrund der anatomischen Nähe zu den großen Gefäßen kann die Entfernung von Osteosynthesematerial oder Knochenspänen von ventral besonders kritisch werden.

\section{Operativer Workflow}

Bei thorakoskopischer Instrumentierung muss im Rahmen der Narkose eine Intubation mit einem Doppellumentubus erfolgen, um intraoperativ die Lunge der 


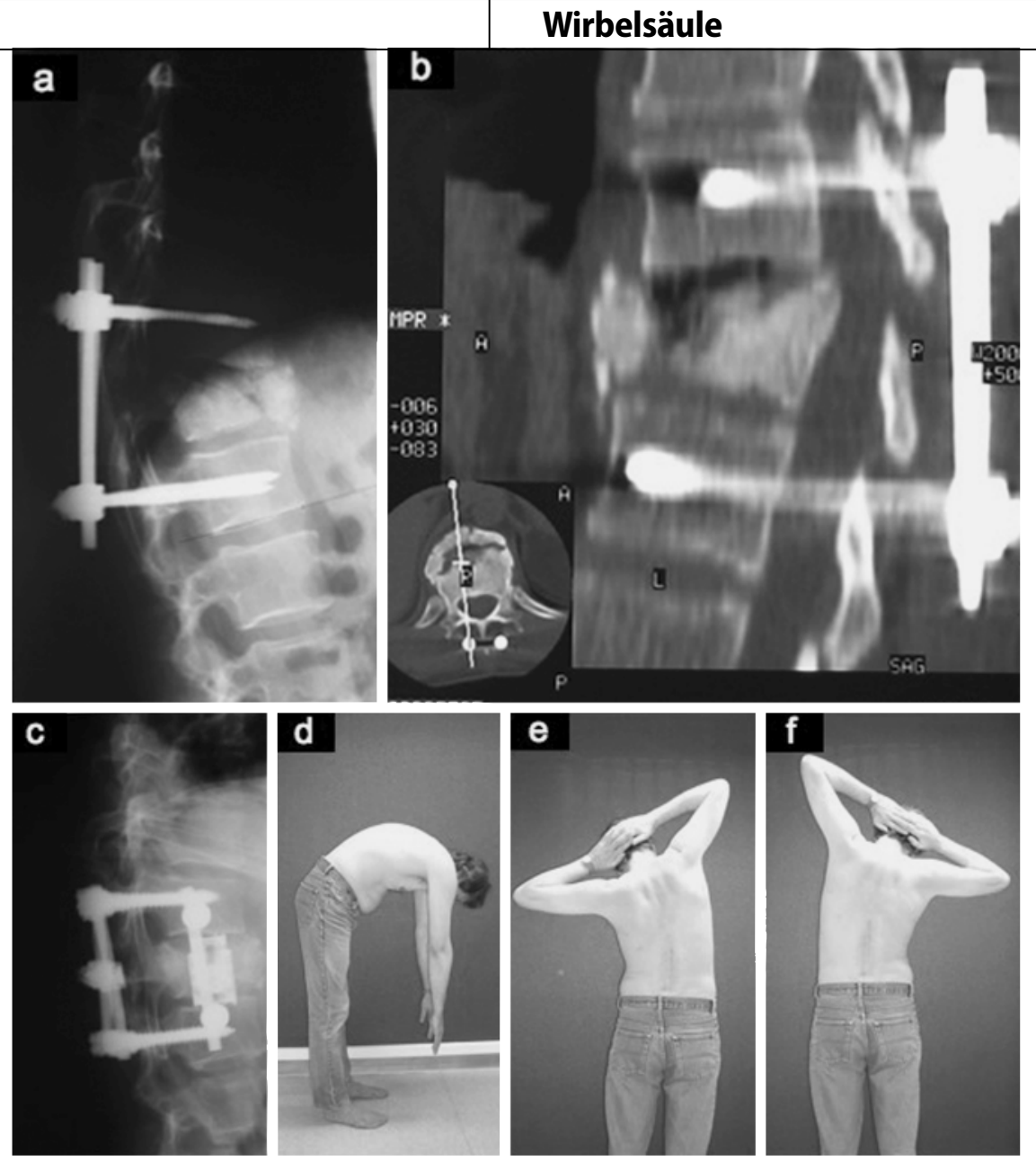

Abb. 5 \ 41 Jahre alter männlicher Patient, 5 Monate nach Trauma (frontaler Spaltbruch Th12 - AO A2.2), a,b sekundäre Kyphose nach alleiniger dorsaler Instrumentierung (GDW $\left.20^{\circ}\right)$, c bisegmentale dorsoventrale Korrekturspondylodese, GDW $6^{\circ}$, $\mathbf{d - f}$ gutes funktionelles Ergebnis 26 Monate nach Korrektureingriff

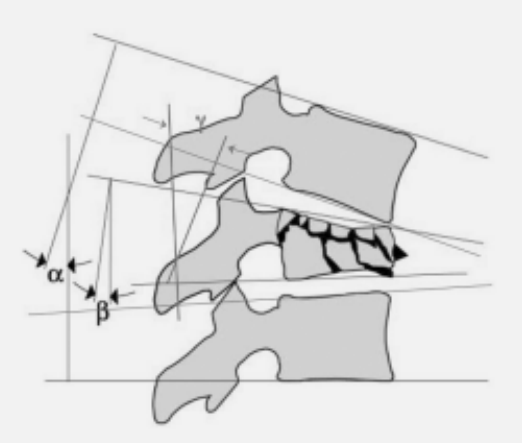

Abb. $6 \triangle$ Schematische Darstellung zur Messung der vertebralen $(\beta)$, bisegmentalen $(\alpha)$ und monosegmentalen $(\gamma)$ Kyphosewinkel (GDW), modifiziert nach Eysel et al. [6]

betroffenen Zugangsseite kollabieren lassen zu können. Die korrekte Platzierung des Tubus sollte fieberoptisch über ein Kinderbronchoskop kontrolliert werden.

Bei Fehlstellungen des Gefüges ist eine intraoperative Mobilisation des be-

troffenen Segments erforderlich. Dazu sind umständliche intraoperative Manöver mit evtl. Entfernung des dorsalen Osteosynthesematerials und Arthrolyse der dorsalen Zwischenwirbelgelenke notwendig. Gelegentlich müssen die kleinen Gelenke reseziert oder dorsale Spondylodesen osteotomiert werden.

Die dorsale Instrumentierung wird in Bauchlage durchgeführt. Eventuell muss erst eine Materialentfernung nicht korrekt platzierter oder gebrochener Pedikelschrauben erfolgen. Für die Neuverankerung können aufgrund von ausgelockerten Pedikelschrauben und dadurch bedingten Osteolysen besondere Probleme resultieren.

Prinzipiell kann die ventrale Instrumentierung entweder in Bauchlage, minimalinvasiv, endoskopisch assistiert über eine Minithorakotomie und Einsatz von Retraktorsystemen [20], in Sei- tenlagerung, ebenfalls endoskopisch assistiert, transthorakal oder konventionell offen erfolgen.

Über den transthorakalen Zugang können von links (Standardzugang) die Segmente Thy bis etwa L2 erreicht werden, von rechts etwa Th4-L1 [20]. Alternativ kann der Zugang auch retroperitoneal (ab LWK2), in der Regel minimalinvasiv und endoskopisch gestützt, erfolgen. In jedem Fall sollte sowohl instrumentell als auch personell (Gefäßchirurg) die sofortige Bereitschaft zu einer Thorakotomie gegeben sein.

Im Rahmen der ventralen Instrumentierung erfolgen in Abhängigkeit von der Fraktur die mono- oder bisegmentale Spondylodese mit Entfernung des Knochendefekts im betroffenen Wirbelkörper, der kranialen und/oder kaudalen Bandscheibe, die Mobilisation der ventralen Säule und der Ersatz der ventralen Säule durch autologen trikortikalen Knochenspan, Spongiosa oder durch $\mathrm{Ca}-$ ge-Systeme als Wirbelkörpersatz. Kombiniert können auch ein ventrales, unilaterales Stabsystem oder winkelstabiles Plattensystem zum Einsatz kommen. Eine Spongiosaplastik ist auch bei Verwendung eines Cage-Systems durchzuführen ( $\bullet$ Abb. 7). Ziel dieser ventralen Rekonstruktion und Abstützung sollten eine dauerhafte Stabilität der Achskorrektur und ein möglichst geringer sekundärer Korrekturverlust sein [12]. Sie dienen der Entlastung der ergänzenden dorsalen, zuggurtenden Stabilisierung [10].

Nach abgeschlossener ventraler Instrumentierung und dem Einbringen der Spongiosa schließt die endgültige Implantation der dorsalen Fixateursystemstäbe den Eingriff ab.

\section{Prognose}

Objektiv abschätzbare prognostische Kriterien sind das Ausmaß der erzielten Achskorrektur, die Suffizienz der Spondylodese im Sinne einer korrekten Platzierung der Pedikelschrauben sowie die korrekte ventrale Abstützung. Ein vollständiges Einwachsen der ventralen Abstützsysteme ist langfristig die Voraussetzung für einen möglichst geringen Korrekturverlust und damit für eine Minimierung von Folgeschäden. 

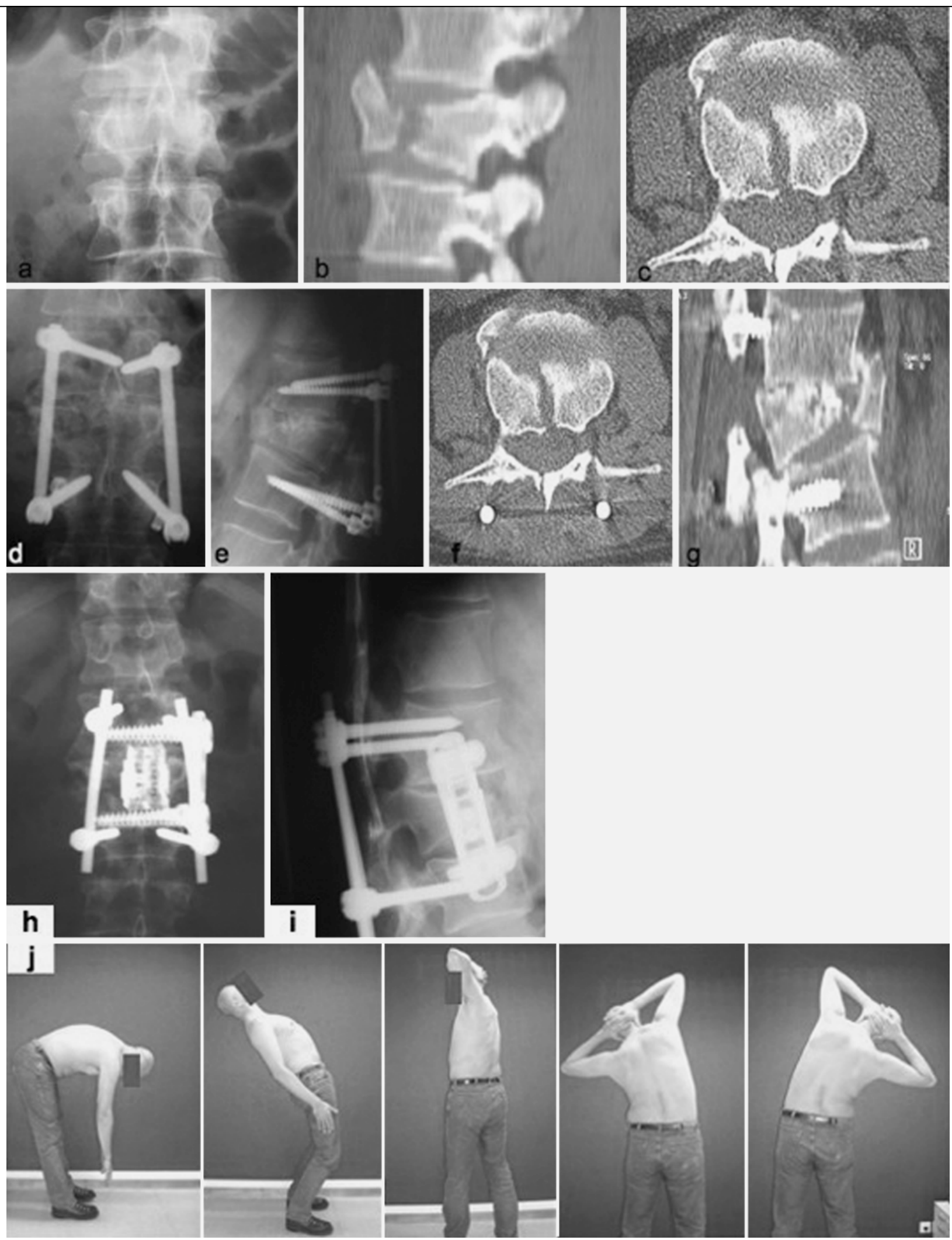

Abb. 7 \ 43 Jahre alter Patient mit Kneifzangenberstungsbruch LWK2 (AO A3.3.1) nach Motorradsturz, keine neurologischen Ausfälle, dorsale Spondylodese mit Fixateur interne 15 Tage nach Unfall, a-c Unfallbilder, d-g Bilder vor Korrektureingriff (5 1/2 Monate nach Erstversorgung): deutlicher Korrekturverlust und Ausbildung einer posttraumatischen Kyphose (keine Neurologie), h,i postoperativer Zustand nach Entfernung des dorsalen Fixateur interne und kombinierter dorsoventraler Korrekturspondylodese: dorsale Respondylodese von L1 auf L3 und ventrale, endoskopisch assistierte Korporektomie, Stabilisierung über expandierbarem Titancage und winkelstabilem Titanplattensystem, $\mathbf{j}$ sehr gutes funktionelles Ergebnis bei der Nachuntersuchung 1 Jahr nach Korrektureingriff 


\section{Ergebnisse}

Es gibt nur wenige Arbeiten, die über Ergebnisse nach dorsoventral instrumentierten Korrekturspondylodesen im thorakolumbalen Übergang berichten $[9,16$, 17].

Eine Besserung neurologischer Ausfälle nach Korrekturoperation wird in der Literatur bei $53-64 \%$ der Patienten beschrieben, im Sinn einer Verbesserung der Symptome auf der Frankel-Skala [9, 16, 17]. Hierbei sind Verbesserungen von einer und mehreren Stufen berücksichtigt.

Die Differenz der unmittelbar präoperativ sowie unmittelbar postoperativ gemessenen GDW (s. oben) spiegelt die Reposition der Fehlstellung wider. Die Repositionsergebnisse betragen durchschnittlich $13,0^{\circ}$ bei konservativ und $12,3^{\circ}$ bei operativ vorbehandelten Patienten [9]. Suk et al. [17] berichteten über eine durchschnittliche Reposition von $11,8^{\circ}$ bei dorsoventralem Vorgehen und Stolze u. Harms [16] von einer „exakten und profilgerechten“ Reposition in 93\% der Fälle.

Der Korrekturverlust, nachgemessen an der Differenz des GDW unmittelbar postoperativ nach Korrekturspondylodesen und zum Nachuntersuchungszeitpunkt, weist in der Literatur große Spannbreiten auf und wird mit $2,7-30,7^{\circ}$ angegeben $[9,16,17]$.

Eine signifikante Besserung der subjektiven Schmerzsymptomatik konnte bei bis zu 81\% der operierten Patienten erzielt werden [16].

\section{Fazit für die Praxis}

Zur Prävention posttraumatischer Fehlstellungen gehören eine adäquate Diagnostik und die korrekte Indikationsstellung zur konservativen oder operativen Therapie.

Die symptomatische kyphotische Fehlstellung des thorakolumbalen Übergangs mit einem Kyphosewinkel von $>20^{\circ}$ ergibt die Indikation für den Eingriff. Der Korrektureingriff bedarf einer exakten präoperativen Planung und sollte in der Regel kombiniert dorsoventral instrumentiert werden.

Bis zu $81 \%$ der operierten Patienten erfahren eine signifikante Besserung der
Schmerzsymptomatik. Je nach Autor beträgt der Korrekturverlust bis zu 30,7. Aufgrund der anspruchsvollen Operationstechnik und der evtl. Notwendigkeit zur interdisziplinären Zusammenarbeit sollten diese Korrektureingriffe Wirbelsäulenzentren vorbehalten bleiben.

\section{Korrespondierender Autor}

Prof. Dr. H. Lill

Klinik für Unfall- und Wiederherstellungschirurgie, Friederikenstift Hannover Humboldtstraße 5, 30169 Hannover helmut.lill@friederikenstift.de

Interessenkonflikt. Es besteht kein Interessenkonflikt. Der korrespondierende Autor versichert, dass keine Verbindungen mit einer Firma, deren Produkt in dem Artikel genannt ist, oder einer Firma, die ein Konkurrenzprodukt vertreibt, bestehen. Die Präsentation des Themas ist unabhängig und die Darstellung der Inhalte produktneutral.

\section{Literatur}

1. Blauth M, Knop C, Bastian L et al. (1997) Neue Entwicklungen in der Chirurgie der verletzten Wirbelsäule. Orthopäde 26: 437-449

2. Blauth M, Knop C, Bastian L (1998) Brust- und Lendenwirbelsäule. In: Tscherne H, Blauth M (Hrsg) Tscherne Unfallchirurgie - Wirbelsäule. Springer, Berlin Heidelberg New York, S 241-372

3. Böhler L (1972) Konservative Behandlung von Brüchen der Brust- und Lendenwirbelsäule. Unfallmed Berufserkrank 2: 100-104

4. Bohlmann HH (1976) Late progressive paralysis and pain following fracture of the thoracolumbar spine. J Bone Joint Surg Am 58: 728

5. Bradford DS, McBride GG (1987) Surgical management of thoracolumbar spine fractures with incomplete neurological deficits. Clin Orthop 218: 882-900

6. Eysel P, Hopf C, Fürderer S (2001) Kyphotische Deformierungen bei Frakturen der Brust- und Lendenwirbelsäule. Orthopäde 30: 955-964

7. Feil R, Wich M, Ekkenkamp A (2003) Korrektureingriffe nach BWS- und LWS-Verletzugen. Trauma Berufskrankh 5: 342-346

8. Katscher S, Verheyden P, Gonschorek O et al. (2003) Thorakolumbale Wirbelfrakturen nach konservativer und operativer Behandlung. Unfallchirurg 106: 20-27

9. Klöckner C, Hofmann A, Weber U (2001) Die posttraumatische Kyphose der Rumpfwirbelsäule. Orthopäde 30: 947-954

10. Knop C, Blauth M, Bastian L et al. (1997) Frakturen der thorakolumbalen Wirbelsäule. Unfallchirurg 100: 1032-1047

11. Knop C, Blauth M, Bühren V et al. (2000) Operative Behandlung von Verletzungen des thorakolumbalen Übergangs.Teil 2: Operationen und röntgenologische Befunde. Unfallchirurg 103: 1032-1047

12. Knop C, Blauth M, Bühren V et al. (2001) Behandlung von Verletzungen des thorakolumbalen Übergangs. Teil 3: Nachuntersuchung. Unfallchirurg 104: 583-600
13. Lange U, Bastian L, Knop C et al. (2002) Einfluss einer kurzstreckigen Spondylodese des thorakolumbalen Übergangs auf die angrenzenden Wirbelgelenke. Unfallchirurg 105: 359-370

14. Marré B (2005) Management of posttraumatic kyphosis: surgical technique to facilitate a combined approach. Injury 36: S-B73-S-B81

15. Pierce DS (1969) Spinal cord injury with anterior decompression, fusion and stabilization and early rehabilitation. J Bone Joint Surg Am 51: 1675

16. Stolze D, Harms J (1999) Korrekturen posttraumatischer Fehlstellungen. Orthopäde 28: 731-745

17. Suk S, Kim JH, Lee SM et al. (2003) Anterior-posterior surgery versus posterior closing wedge osteotomy in posttraumatic kyphosis with neurologic compromised osteoporotic fracture. Spine 28: 2170-2175

18. Trojan E (1972) Langfristige Ergebnisse von 200 Wirbelbrüchen der Brust- und Lendenwirbelsäule ohne Lähmungen. Unfallmed Berufskrankh 66: 122-134

19. Vaccaro AR, Silber JS (2001) Post-traumatic spinal deformity. Spine 26: S111-S118

20. Verheyden AP, Katscher S, Gonschorek S et al. (2002) Endoskopisch assistierte Rekonstruktion der thorakolumbalen Wirbelsäule in Bauchlage. Unfallchirurg 105: 873-880 